\title{
Revisión bibliográfica: Efectos del ejercicio en la presión intraocular
}

\author{
Alemán Ramírez, Carolina \\ Revisión bibliográfica: Efectos del ejercicio en la presión intraocular \\ MHSalud, vol. 16, núm. 2, 2019 \\ Universidad Nacional, Costa Rica \\ Disponible en: http://www.redalyc.org/articulo.oa?id=237059549001 \\ DOI: https://doi.org/10.15359/mhs.16-2.1
}

Esta obra está bajo una Licencia Creative Commons Atribución-NoComercial-SinDerivar 3.0 Internacional. 


\title{
Revisión bibliográfica: Efectos del ejercicio en la presión intraocular
}

\author{
Literature Review: Effects of Exercise on Intraocular Pressure \\ Revisão da literatura: efeitos do exercício na pressão intra-ocular
}

Carolina Alemán Ramirez

Universidad Nacional, Costa Rica

caroaleman@gmail.com

(iD http://orcid.org/0000-0001-9408-2366
DOI: https://doi.org/10.15359/mhs.16-2.1

Redalyc: http://www.redalyc.org/articulo.oa?

$\mathrm{id}=237059549001$

Recepción: 26 Septiembre 2018

Aprobación: 15 Mayo 2019

\section{RESUMEN:}

Introducción: La relación entre la presión intraocular (PIO) y el ejercicio agudo se ha venido estudiando en los últimos años. Se ha encontrado que el ejercicio agudo tiene un efecto positivo en la PIO; sin embargo, el ejercicio aeróbico y el ejercicio contra resistencia presentan resultados diferentes. En cuanto al ejercicio aeróbico agudo, brinda un efecto positivo en la PIO, ya que esta disminuye. Mientras que el ejercicio contra resistencia presenta una variabilidad en los resultados obtenidos de acuerdo con las investigaciones consultadas. Propósito: Mostrar los resultados obtenidos de las investigaciones relacionadas con el ejercicio aeróbico y el ejercicio contra resistencia de forma aguda en la presión intraocular, para así poder brindar recomendaciones a poblaciones sanas y con glaucoma. Método: Revisión de bibliografía, búsqueda de artículos científicos en las siguientes bases de datos: SportDiscus con texto completo, psycINFO, Psychology and Behavioral Sciences Collection, Rehabilitation \& Sports Medicine Source, Google Académico, Research Gate, PubMed. Resultados: El ejercicio aeróbico agudo pareciera tener un efecto significativo sobre la presión intraocular, pues la disminuye. Este resultado se presenta en personas sedentarias y con glaucoma. Para personas físicamente activas o atletas, para lograr reducir la PIO es necesario realizar ejercicio a intensidades más altas, comparado con personas sedentarias. Con respecto a la duración de ejercicio aeróbico agudo, parece que con solo cinco minutos se produce una disminución sobre la PIO. Al finalizar el ejercicio, el efecto agudo del ejercicio aeróbico se mantiene por un corto tiempo sobre la PIO. Son necesarias más investigaciones de efecto crónico. Igualmente, se necesitan más estudios que involucren variables como sexo y edad dentro de los análisis y no como estadística descriptiva, para así conocer el efecto del ejercicio sobre estas mismas. Con respecto a los ejercicios de contra resistencia, la parte superior del cuerpo aumenta más la PIO, al compararlos con ejercicios de la parte inferior. Los ejercicios de contra resistencia de alta intensidad aumentan la PIO. Conclusiones: Los resultados demuestran que las personas con condición visual normal y con glaucoma pueden realizar ejercicio aeróbico y logran un efecto positivo en su salud visual. Por otro lado, debido a la falta de investigación en ejercicio de contra resistencia, se recomienda que se realicen estudios con intensidades bajas, según los datos obtenidos en esta revisión. Con respecto a la calidad de los estudios encontrados podrían mejorarse los diseños en las investigaciones: la mayoría no contó con grupos controles, tampoco especificaron la intensidad del ejercicio e incluso les faltó incluir aspectos descriptivos necesarios para poder contar con información más clara.

Palabras clave: Presión intraocular, glaucoma, ejercicio aeróbico, ejercicio contra resistencia, actividad física.

\section{Abstract:}

Introduction: The relationship between intraocular pressure (IOP) and acute exercise has been studied in recent years. It has been found that acute exercise has a positive effect on IOP; however, aerobic exercise and strength exercise present different results. As for acute aerobic exercise, it can be mentioned that it provides a positive effect on the intraocular pressure since it decreases, while the strength exercise gives variability in the results obtained according to the research studies consulted. Purpose: Show the results obtained from the investigation related to the aerobic exercise and strength exercise and intraocular pressure in order to provide recommendations to healthy populations with glaucoma. Methods: The literature review searched for articles in the following databases: SportDiscus with complete text, PsycINFO, Psychology and Behavioral Sciences Collection, Rehabilitation \& Sports Medicine Source, Google academics, Research Gate, PubMed. Results: Acute aerobic exercise seems to have a significant effect on intraocular pressure: it decreases it. This result occurs in sedentary people with glaucoma. For physically active people or athletes, in order to reduce IOP, it is necessary to exercise at higher intensities compared to sedentary people. Regarding the length of acute aerobic exercise, it seems that, with only 5 minutes, there is a decrease in IOP. At the end of the exercise, the acute effect of aerobic exercise seems to be maintained for a short time in the IOP; more research on the chronic effect is necessary. Also, more research is needed and should involve variables, such as sex and age, within the study analyses and not as descriptive statistics, in order to know the effect of exercise on them. Regarding the strength exercises, the IOP increases in the upper part of the body when compared with exercises in the lower part. High-intensity strength exercises increase the IOP. Conclusions: The results show that people 
with normal visual condition and glaucoma can perform aerobic exercise, achieving a positive effect on their visual health. On the other hand, there is a lack of research in strength exercise; it is recommended that these studies be conducted at low intensities, according to the data obtained in this review. Regarding the quality of the studies found, the designs could be improved in the investigations. Most of these studies did not have control groups, nor did they specify the intensity; besides, they missed including descriptive aspects needed to be able to have more accurate information.

KEYWORDS: intraocular pressure, physical activity, exercise, glaucoma.

\section{Resumo:}

Introdução A relação entre a pressão intraocular (PIO) e o exercício agudo foi estudada nos últimos anos. Descobrindo que o exercício agudo tem um efeito positivo na PIO, no entanto, o exercício aeróbico e o exercício de resistência têm resultados diferentes. No que diz respeito ao exercício aeróbico agudo, pode mencionar-se que tem um efeito positivo na PIO, uma vez que esta diminui. Enquanto o exercício de resistência dá uma variabilidade nos resultados obtidos de acordo com as pesquisas consultadas. Objetivo: Mostrar os resultados obtidos em pesquisas relacionadas ao exercício aeróbio e ao exercício de resistência aguda na pressão intraocular, a fim de fornecer recomendações para populações saudáveis e com glaucoma. Métodos: revisão de literatura, busca de artigos científicos nas seguintes bases de dados: SportDiscus com texto completo, psycINFO, Psychology and Behavioral Sciences Collection, Rehabilitation \& Sports Medicine Source, Google Academic, Research Gate, PubMed. Resultados: O exercício aeróbio agudo parece ter um efeito significativo em diminuir a pressão intraocular, este resultado ocorre em pessoas sedentárias com glaucoma. Para pessoas fisicamente ativas ou atletas parece que, para reduzir a PIO é necessário se exercitar em maiores intensidades, em comparação com pessoas sedentárias. Com relação à duração do exercício aeróbico agudo, parece que, com apenas cinco minutos, há uma diminuição da PIO. No final do exercício, o efeito agudo do exercício aeróbico parece ser mantido por um curto período de tempo na PIO, sendo necessária mais pesquisa de efeitos crônicos. Da mesma forma, são necessárias mais pesquisas envolvendo variáveis como sexo e idade dentro da análise do estudo e não como estatística descritiva, com o fim de conhecer o efeito do exercício sobre essas variáveis. Com relação aos exercícios de resistência, a parte superior do corpo aumenta mais a PIO comparada aos exercícios da parte inferior do corpo. Exercícios de resistência de alta intensidade aumentam a PIO. Conclusões: Os resultados mostram que pessoas com visão normal e glaucoma podem realizar exercícios aeróbicos, obtendo um efeito positivo na sua saúde visual. Por outro lado, há uma falta de pesquisas sobre o exercício de resistência, sendo recomendável que esses estudos sejam realizados com baixas intensidades, de acordo com os dados obtidos nesta revisão. Com relação à qualidade dos estudos encontrados, os desenhos de pesquisa puderam ser melhorados, a maioria não possuía grupos controle, nem especificavam a intensidade do exercício, e ainda faltavam os aspectos descritivos necessários para poder ter informações mais claras.

Palavras-CHAVE: pressão intraocular, glaucoma, exercício aeróbio, exercício de resistência, atividade física.

\section{INTRODUCCIÓN}

La salud es uno de los conceptos de bienestar integral más importantes en el ser humano, por lo tanto, la salud visual constituye parte de ese bienestar. A nivel mundial hay 253 millones de personas con discapacidad visual, de las cuales 36 millones presentan una condición de ceguera y 217 millones baja visión (OMS, s.f.). En nuestro país, el 5,85 \% de la población tiene discapacidad visual, aunque no se conocen los datos en porcentajes por diagnóstico visual (cataratas, retinosis pigmentaria, glaucoma, etc.) es la discapacidad con mayor prevalencia, es decir, la que abarca el porcentaje más alto de la población (Instituto Nacional de Estadística y Censos [Costa Rica], 2014). Uno de los aspectos que representa un factor negativo para la salud visual es la presión intraocular alta. La presión intraocular, también llamada PIO, es la que ejercen los líquidos intraoculares o bien el humor acuoso contra la pared anterior del ojo, la cual es necesaria para que este órgano se mantenga distendido (Zhu et al., 2018). Por esta razón, se hace necesario una revisión bibliográfica para conocer los beneficios del ejercicio físico que eventualmente podría ejercer efectos en la PIO.

\section{Propósito}

El propósito de esta revisión bibliográfica es la de mostrar los resultados obtenidos de las investigaciones relacionadas con el ejercicio aeróbico y el ejercicio contra resistencia de forma aguda en la presión intraocular, para así poder brindar recomendaciones a poblaciones sanas y con glaucoma. 


\section{ANTECEDENTES TEÓRICOS}

Para mantener un apropiado funcionamiento visual del ojo, es necesario que sus sistemas se encuentren bien. Un ejemplo de esto es la PIO; para que esta se mantenga equilibrada, debe darse un balance entre la producción y el drenaje del humor acuoso (Ozmerdivenli, Simsek, Bulut, Karacabey, \& Saygin, 2006) . El humor acuoso es un líquido que se encuentra en el globo ocular, es secretado por el cuerpo ciliar, ubicado posterior al cristalino. Este líquido atraviesa la pupila con dirección a la cámara anterior, donde se filtra en la malla trabecular y el canal de "Schlemm" antes de drenar al sistema venoso. El humor acuoso cumple con dos funciones muy importantes: la primera es determinar la PIO, por lo que una obstrucción de salida aumentará la PIO; la segunda es reemplazar a la sangre en la nutrición de la córnea y del cristalino; también distribuye nutrientes esenciales para estos tejidos y recoge productos de desecho. Así mismo, el humor acuoso carece de células, pigmentos y proteínas que tiene la sangre, por lo tanto, facilita el paso de luz (Yan et al., 2016) . La elevación de PIO no solo lleva a la compresión mecánica del nervio óptico, sino también a la restricción del suministro de sangre a este.

Por otro lado, los valores normales de la PIO deben oscilar entre 11 mm Hg y $21 \mathrm{~mm} \mathrm{Hg}$ (Roddy, 2014). Estos valores se pueden obtener por medio de una tonometría, método utilizado con mucha frecuencia para conocer los niveles de presión intraocular. Una PIO elevada representa un factor de riesgo modificable que se puede y debe controlar, para prevenir el desarrollo o progreso del glaucoma (Aspinall et al., 2008; Era, Pärssinen, Kallinen, \& Suominen, 2009; Leighton, 1972; Zhu et al., 2018) .

Ahora bien, la actividad física y el ejercicio, sin lugar a duda, tienen un papel determinante en la calidad de vida de las personas (Aspinall et al., 2008; Avunduk, Yilmaz, Şahin, Kapicioglu, \& Dayanır, 1999; Chong et al., 2016; Kinoshita et al., 2016), porque mejora parámetros de salud importantes, y también constituye un factor de protección y prevención de enfermedades crónicas (Roddy, 2014).

Otro aspecto importante del ejercicio y de la actividad física es que brindan beneficios, como un efecto crónico, no solo después de varias sesiones de ejercicio, sino también con una sola sesión con un efecto agudo (Dane, KoçEr, Demirel, üçOk, \& Tan, 2006; Era et al., 2009; Karabatakis et al., 2004; Ozmerdivenli et al., 2006; Vera, García-Ramos, Jiménez, \& Cárdenas, 2017).

\section{EJERCICIO AERóbICO Y PIO}

Con respecto a los efectos de ejercicio o actividad física sobre los cambios en la PIO, se han encontrado en la bibliografía resultados inconsistentes en algunas variables como el tipo de ejercicio, la intensidad y la duración ( Martin, Harris, Hammel, \& Malinovsky, 1999; Bakke, Hisdal, \& Semb, 2009; Read \& Collins, 2011; Huang, 2015; Vera et al., 2017) . En cuanto al ejercicio aeróbico realizado de forma aguda, ya sea caminar, trotar, correr o andar en bicicleta, se ha determinado una disminución de la PIO; este resultado se ha observado con sesiones que van desde los cinco minutos de duración hasta una hora, también la PIO ha disminuido con diferentes intensidades de ejercicio (Conte \& Scarpi, 2014; Dane et al., 2006; Huang, 2015; Soares et al., 2015; Vera et al., 2017; Vieira, 2006).

El estudio realizado por Dane et al. (2006) tuvo dos poblaciones de sujetos: un grupo de personas sedentarias y otro de atletas. Las personas sedentarias lograron disminuir la PIO $1.72 \mathrm{~mm} \mathrm{Hg}$, después de realizar un trote de cinco minutos al 70\% de VO2max. Sin embargo, el grupo de atletas que participó en el estudio no logró ningún cambio en la PIO. Mencionan que el estado físico de las personas podría ser un factor que determine el cambio agudo de la PIO, por lo que las personas físicamente activas o atletas deberían hacer ejercicio aeróbico a una intensidad más alta para lograr reducciones. Ahora bien, el equipo investigativo no mencionan una recomendación de intensidad para que sujetos atletas también logren estas reducciones.

En la misma línea, el estudio de Ozmerdivenli et al. (2006) compara los cambios de la PIO después de correr 30 minutos al $70 \%$ del VO2max. en personas activas y sedentarias. Las personas activas disminuyeron 
$2.92 \mathrm{mmHg}$ y las personas sedentarias disminuyeron $4.64 \mathrm{mmHg}$, lo que demostró que el ejercicio agudo redujo más la PIO en personas sedentarias que en activas.

Harris, Malinovsky, \& Martinx (1994) muestran que las personas activas reducen la PIO en $4.7 \mathrm{mmHg}$ y las personas acostumbradas a un sistema de entrenamiento más vigoroso y habitual a $2.6 \mathrm{mmHg}$, al realizar ejercicio en un cicloergómetro durante 10 minutos con una resistencia de 90 watts. Mencionan que la intensidad debe ser media y alta para lograr cambio en la PIO.

Se observa la misma tendencia en los resultados de estos estudios, es decir, la condición física es un factor que determina el cambio, lo que pareciera que cuanto más actividad física intensa y frecuente realice una persona, las reducciones de la PIO van a ser menores (Dane et al., 2006; Harris et al., 1994; Ozmerdivenli et al., 2006).

Ahmad (1995), en su estudio, comparó la intensidad del ejercicio en tres condiciones: caminar, trotar y correr. Encontró que las personas que trotaron redujeron más la PIO que las que caminaron y, a su vez, quienes corrieron redujeron más la PIO que quienes trotaron, es decir, a mayor intensidad, mayor disminución de le PIO. También encontró que las personas que tenían un diagnóstico visual de glaucoma mostraron la misma tendencia que las personas con visión normal, es decir, que a mayor intensidad de ejercicio mayor reducción. Las personas con glaucoma lograron una reducción de $7,72 \mathrm{~mm} \mathrm{Hg}$ después de caminar, de10,86 $\mathrm{mmHg}$ después de trotar y de 13,85 $\mathrm{mmHg}$ después de correr; las personas de de visión normal redujeron 2,43 $\mathrm{mm} \mathrm{Hg}$ después de caminar, de 3,85 $\mathrm{mm}$ Hg después de trotar y de $4 \mathrm{~mm} \mathrm{Hg}$ después de correr, por lo que estos datos muestran también que a mayor nivel de PIO inicial (personas con glaucoma), mayor reducción al finalizar el ejercicio. Este estudio concluyó tres aspectos relevantes: una elevada PIO inicial va a lograr una mayor disminución; a más alta intensidad de ejercicio se logra una mayor reducción de la PIO y tanto pacientes con glaucoma como pacientes con visión normal la reducen.

Karabatakis et al. (2004) realizaron un estudio con personas sanas, para conocer los cambios de la PIO después de trotar durante 20 minutos al $70 \%$ de la frecuencia cardiaca máxima (FC max.). Se encontró una reducción de $2,73 \mathrm{~mm} \mathrm{Hg}$ en el ojo derecho y 2,94 $\mathrm{mm} \mathrm{Hg}$ en el ojo izquierdo. Mencionan que no encontraron diferencias en la disminución de la PIO entre ambos ojos, también indican que lo más influyente en la reducción de la PIO es la intensidad del ejercicio.

Yan et al. (2006) lograron una disminución de $4.3 \mathrm{~mm} \mathrm{Hm}$, después de trotar por 20 minutos al $70 \%$ frecuencia cardiaca máxima, en jóvenes saludables.

Ahmad et al. (1996) realizaron un protocolo con diferentes intensidades, para examinar la reducción de la PIO en hombres sedentarios. Se realizó ejercicio en un cicloergómetro durante 15 minutos, cuando la intensidad fue al 80 \% FC max. se redujo en $4.7 \mathrm{mmHg}$, al 60 \% FC max. disminuyó en 3,5 mmHg y al $40 \%$ FC max. se redujo en $0,9 \mathrm{mmHg}$. Igualmente, que otros estudios, concluyen que la intensidad del ejercicio es responsable de la magnitud de las disminuciones de la PIO.

Por lo tanto, la intensidad del ejercicio tiene un papel fundamental para lograr reducciones significativas en la presión intraocular. Por lo tanto, tal y como se mostró en la información anteriormente citada, baja intensidad de ejercicio logra reducciones en la PIO, pero intensidades más altas, logran mayor cambio (Karabatakis et al., 2004; Ozmerdivenli et al., 2006; Read \& Collins, 2011; Yan et al., 2016; Ahmad 1995; Amnad., 1996; Rüfer et al., 2014).

Otro aspecto observable consiste en que cuando el ejercicio se finaliza, los valores de la PIO regresan a su rango normal, en un periodo de corto tiempo (Kozobolis, Detorakis, Konstas, Achtaropoulos, \& Diamandides, 2008).

Okuno, Sugiyama, Kohyma, Kojima, Oku, \& Ikeda (2006) afirman este aspecto. El protocolo de ejercicio aeróbico consistió en realizar durante una hora intervalos, con "steps", después de finalizar el ejercicio la normalización de la PIO se dio en una hora.

Así mismo, Red and Collins. (2011) reportaron reducciones de $1.71 \mathrm{mmHm}$ después de 10 minutos de ejercicio en un cicloergómetro. Ellos encontraron también que los valores iniciales de la PIO se estabilizaron 
después de 10 minutos de finalizado el ejercicio. Además, concluyen que ejercicios de corta duración logran reducciones en la PIO.

En el estudio de Esfahani, Gharipour, \& Fesharakinia (2017), se muestra esta tendencia: las personas después de caminar cinco minutos (no especificaron intensidad), disminuyen significativamente la PIO en $2.33 \mathrm{mmHg}$; y con 20 minutos de descanso se recobran los valores iniciales. También correlacionaronn el comportamiento de la $\mathrm{PIO}$ entre hombres y mujeres y no encontraron diferencias significativas entre sexos. En esta investigación se encontró otro dato interesante, cuanta mayor edad, la PIO disminuye de forma más pronunciada, pocas investigaciones hacen este tipo de análisis.

Para Yan et al. (2016) y Ahmad (1995), algunas de las razones por las que el ejercicio aeróbico agudo podría ayudar a disminuir la PIO son: al realizar ejercicio se produce sudor, con esta pérdida de agua, aumenta la presión osmótica del plasma y esto podría disminuir la producción de humor acuoso. Otro aspecto que podría suceder es que el ejercicio facilite el drenaje del humor acuoso. De igual manera, el ejercicio hace que la sangre se concentre en los músculos activos, por lo que reduce el flujo sanguíneo ocular y disminuye la producción de humor acuoso. Por otro lado, el sistema nervioso simpático causa vasoconstricción coroidea, por lo que se reduce el flujo de sangre y disminuye la PIO. También, al darse un incremento de catecolaminas, se disminuye la formación de humor acuoso. Además, podría deberse a la activación de la AMPc la cual hace que la PIO disminuya por la baja producción del humor acuoso.

El ejercicio aeróbico tiene un impacto positivo en los parámetros de la PIO, en poblaciones con diferentes niveles de actividad física, condición visual y condición física (Read \& Collins, 2011).

A continuación, en la Tabla 1, se muestra el resumen de los estudios relacionados con el ejercicio aeróbico agudo y su efecto en la presión intraocular. 
TABLA 1.

Cambios de la PIO con ejercicio agudo aeróbico en diferentes poblaciones

\begin{tabular}{|c|c|c|c|c|c|}
\hline Referencias & Población & Tipo ejercicio aeróbico & Duración & Intensidad & Cambio en PIO \\
\hline \multirow[t]{2}{*}{ Dane et al., 2006} & Sedentaria & Trotar & $5 \mathrm{~min}$ & $70 \% \mathrm{vo}^{2} \max$. & $\downarrow 1,84 \mathrm{~mm} \mathrm{Hg}$ \\
\hline & Atletas & Trotar & $6 \min$ & $70 \% \mathrm{vo}^{2} \max$. & mantuvo \\
\hline \multirow[t]{2}{*}{ Ozmerdivenli, 2006} & Activa & Correr & $30 \mathrm{~min}$ & $70 \%$ vo $^{2} \max$. & $\downarrow 2,92 \mathrm{~mm} \mathrm{Hg}$ \\
\hline & Sedentaria & & & $70 \% \mathrm{vo}^{2} \max$. & $\downarrow 4,64 \mathrm{~mm} \mathrm{Hg}$ \\
\hline Yan et al., 2016 & Jóvenes saludables & Trotar & $20 \mathrm{~min}$ & $70 \% \mathrm{FC}$. & $\downarrow 4,3 \mathrm{~mm} \mathrm{Hg}$ \\
\hline \multirow[t]{6}{*}{ Ahmad,1995 } & Visión normal & Caminar & $60 \mathrm{~min}$ & NR & $\downarrow 2,43 \mathrm{~mm} \mathrm{Hg}$ \\
\hline & & Trotar & $60 \mathrm{~min}$ & & $\downarrow 3,85 \mathrm{~mm} \mathrm{Hg}$ \\
\hline & & Correr & & & $\downarrow 4 \mathrm{~mm} \mathrm{Hg}$ \\
\hline & Glaucoma & Caminar & $60 \mathrm{~min}$ & & $\downarrow 7,72 \mathrm{~mm} \mathrm{Hg}$ \\
\hline & & Trotar & $60 \mathrm{~min}$ & & $\downarrow 10,86 \mathrm{~mm} \mathrm{Hg}$ \\
\hline & & Correr & & & $\downarrow 12,86 \mathrm{~mm} \mathrm{Hg}$ \\
\hline Red and Collins, 2011 & Adulta & Cicloergómetro & $10 \mathrm{~min}$ & $70 \% \mathrm{FC}$ & $\downarrow 1,71 \mathrm{~mm} \mathrm{Hg}$ \\
\hline Marcus, 1970 & & Correr & $10 \mathrm{~min}$ & Moderado & $\downarrow 5,9 \mathrm{~mm} \mathrm{Hg}$ \\
\hline \multirow[t]{2}{*}{ Harris et al., 1995} & Entrenada & Cicloergómetro & $10 \mathrm{~min}$ & 90 watts & $\downarrow 2,6 \mathrm{~mm} \mathrm{Hg}$ \\
\hline & Activa & Cicloergómetro & $10 \mathrm{~min}$ & 90 watts & $\downarrow 4,7 \mathrm{~mm} \mathrm{Hg}$ \\
\hline Kozobolis, 2008 & & Protocolo de Bruce & NR & NR & $\downarrow 5,65 \mathrm{~mm} \mathrm{Hg}$ \\
\hline \multirow[t]{3}{*}{ Ahmad et al., 1996} & Hombres & Cicloergómetro & $15 \mathrm{~min}$ & $80 \% \mathrm{FC}$ & $\downarrow 4,7 \mathrm{~mm} \mathrm{Hg}$ \\
\hline & Sedentarios & Cicloergómetro & $15 \mathrm{~min}$ & $60 \% \mathrm{FC}$ & $\downarrow 3,5 \mathrm{~mm} \mathrm{Hg}$ \\
\hline & & Cicloergómetro & $15 \mathrm{~min}$ & $40 \% \mathrm{FC}$ & $\downarrow 0,9 \mathrm{~mm} \mathrm{Hg}$ \\
\hline \multirow[t]{2}{*}{ Karabatakis et al., 2004} & Normal & Trote & $20 \mathrm{~min}$ & $70 \% \mathrm{FC}$ & $\downarrow 2,73 \mathrm{OD} \mathrm{mm} \mathrm{Hg}$ \\
\hline & & & & & $\downarrow 2,94 \mathrm{OI} \mathrm{mm} \mathrm{Hg}$ \\
\hline Esfahini et al., 2017 & Normal & Caminar & NR & NR & $\downarrow 2,33 \mathrm{~mm} \mathrm{Hg}$ \\
\hline
\end{tabular}

$\mathrm{NR}=$ No reporta, $\mathrm{OD}=$ ojo derecho, $\mathrm{OI}=$ ojo izquierdo, $\mathrm{FC}=$ frecuencia cardiaca, Vo2 max. $=$ consumo máximo de oxígeno, $\mathrm{mmHg}=$ milímetros de mercurio.

\section{EJERCICIO CONTRA RESISTENCIA Y PIO}

Con respecto al ejercicio de contra resistencia también se ha estudiado su efecto en la PIO. Se presentan los estudios a continuación. 
Vieira (2006) encontró aumentos en la PIO de 4,4 mmHg después de realizar 4 repeticiones al $80 \%$ de una repetición máxima (1RM) de "press" de pecho.

Vera et al. (2017) encontraron, en su estudio, que a mayor intensidad de ejercicio contra resistencia, la PIO aumenta, por lo que en el "jump squat" realizado al $50 \%$ de 1 RM la PIO se mantuvo estable en el pretest y en el postest, mientras que al realizarlo al $60 \%$ de $1 \mathrm{RM}$ se incrementó en $0,71 \mathrm{~mm} \mathrm{Hg}$. Por otro lado el "press" de pecho realizado al $50 \% 1 \mathrm{RM}$ incrementó la PIO en 2,41 mm Hg; del mismo modo se incrementó la PIO al $60 \%$ de 1 RM incrementa en 3,77 mm Hg y finalmente al $100 \%$ de 1 RM se incrementó en 5,41 $\mathrm{mmHg}$. Por lo tanto, se puede observar que los ejercicios del tren superior, aumentan la PIO más que los del tren inferior. Afirman la necesidad de más estudios de fuerza para medir la PIO, en diferentes poblaciones.

Rüfer et al. (2014) presentaron un estudio realizado con personas activas, se compararon ejercicios de contra resistencia para el tren inferior y para el tren superior. Encontraron que los ejercicios para el tren superior incrementan más la PIO que los del tren inferior. En el tren inferior realizaron "leg curl" 20 repeticiones por 1 serie $(20 \times 1)$ al $65 \%$ de IRM, lograron un aumento de $0,2 \mathrm{mmHg}$ no significativo estadísticamente; en el tren superior "butterfly" se realizaron igual número de repeticiones y series, es decir, (20x1) a un $65 \%$ de $1 \mathrm{RM}$ incrementando $0,8 \mathrm{mmHg}$ estadísticamente significativo. Después de 10 minutos de descanso, volvieron a realizar el ejercicio: la intensidad se incrementó al $75 \%$ de 1 RM y se realizaron 10 repeticiones por una serie $(10 \times 1)$. El resultado fue el incremento de la $\mathrm{PIO}$ en $0,3 \mathrm{mmHg}$; pero no estadísticamente significativo. Mencionan que las personas que tienen alta PIO inicial pueden hacer ejercicio de contra resistencia de baja intensidad.

Conte \& Scarpi (2014) realizaron un estudio, en donde aplicaron como tratamiento una rutina de pesas para conocer el cambio en la PIO con respecto a la intensidad y al volumen, se utilizaron dos protocolos de ejercicio: uno consistía en realizar 15 repeticiones por 3 series al $60 \%$ de 1 RM con 30 seg. de descanso, en este grupo se redujo la PIO en $0,48 \mathrm{~mm} \mathrm{Hg}$ y en el otro protocolo se realizó 8 repeticiones por 3 series al 80 $\%$ de 1 RM con $30 \mathrm{seg}$. de descanso, en donde se incrementó la PIO en 0,83 mm Hg. Mencionan que hacen falta más estudios al respecto, porque es necesario entender qué ocurre con el humor acuoso en el momento de la contracción muscular.

Parece que, en el ejercicio contra resistencia, los resultados en relación con la intensidad se relacionan de forma directa con la PIO, es decir, a mayor intensidad de ejercicio mayor incremento de la PIO, por lo que es un aspecto que debe considerarse, ya que podría ser un factor de riesgo especialmente en personas con glaucoma. Otro aspecto a considerar también, es que los ejercicios del tren superior aumentan más la PIO, al compararlos con los del tren inferior (Conte \& Scarpi, 2014; Vera et al., 2017).

Por otra lado, la apnea voluntaria (suspender respiración) pareciera ser una de las razones por las cuales la PIO aumenta durante los ejercicios de contra resistencia, ya que disminuye el retorno venoso, lo que perjudica el drenaje del humor acuoso; a la vez, si a esta suspensión de respiración se le suma la realización de un esfuerzo, aumentando así la presión del tórax y del abdomen (maniobra de Valsalva), aspecto realizado frecuentemente mientras se ejecuta el levantamiento de peso, la PIO incrementará también (Soares et al., 2015).

Se han realizado estudios con tratamientos de fuerza isométrica que han demostrado resultados controversiales. En el caso de Huang (2015), después de 2 minutos de sentadilla isométrica, las personas disminuyeron la PIO -0,8 mmHg. Contrario a este resultado, Bakke et al (2009) encontraron un incremento de 4,1 mm Hg en la PIO, después de realizar dos minutos de fuerza de empuñada. Ello demuestra la necesidad de más estudios en esta área.

En la Tabla 2, se presenta el resumen de los artículos relacionados con los ejercicios de contra resistencia y la presión intraocular. 
TABLA 2.

Cambios de PIO con ejercicio agudo contra resistencia en diferentes poblaciones

\begin{tabular}{|c|c|c|c|c|c|}
\hline Referencias & Población & $\begin{array}{l}\text { Ejercicio de } \\
\text { Contra } \\
\text { Resistencia }\end{array}$ & $\begin{array}{l}\text { Repeticiones } \\
\text { x Series }\end{array}$ & $\begin{array}{c}\text { Intensidad } \\
\text { 1RM }\end{array}$ & $\begin{array}{l}\text { Cambio en } \\
\text { PIO }\end{array}$ \\
\hline \multirow{3}{*}{$\begin{array}{l}\text { Conti y Scarpi, } \\
2013\end{array}$} & \multirow[t]{3}{*}{ Atleta } & \multirow{3}{*}{$\begin{array}{l}\text { Circuito de } 5 \\
\text { ejercicios }\end{array}$} & $15 \times 3$ & $60 \%$ & \multirow{3}{*}{$\begin{array}{c}\downarrow 0,48 \mathrm{~mm} \\
\mathrm{Hg} \\
\uparrow 0,83 \mathrm{~mm} \mathrm{Hg}\end{array}$} \\
\hline & & & $8 \times 3$ & $80 \%$ & \\
\hline & & & & & \\
\hline \multirow[t]{5}{*}{ Vera et al, 2017} & \multirow[t]{5}{*}{ Activa } & Jump Squat & $1 \times 1$ & $50 \%$ & Se mantuvo \\
\hline & & Jump Squat & $1 \times 1$ & $60 \%$ & $\begin{array}{c}\uparrow 0,70 \mathrm{~mm} \\
\mathrm{Hg}\end{array}$ \\
\hline & & Press de pecho & $1 \times 1$ & $50 \%$ & $\uparrow 2,41 \mathrm{~mm} \mathrm{Hg}$ \\
\hline & & Press de pecho & $1 \times 1$ & $60 \%$ & $\uparrow 3,77 \mathrm{~mm} \mathrm{Hg}$ \\
\hline & & Press de pecho & $1 \times 1$ & $100 \%$ & $\begin{array}{c}\uparrow 5,41 \mathrm{~mm} \\
\mathrm{Hg}\end{array}$ \\
\hline \multirow[t]{3}{*}{$\begin{array}{l}\text { Rüfer et al., } \\
2014\end{array}$} & \multirow[t]{3}{*}{ Activa } & \multirow[t]{2}{*}{ Leg Curl } & $20 \times 1$ & $65 \%$ & $\uparrow 0,2 \mathrm{~mm} \mathrm{Hg}$ \\
\hline & & & $10 \times 1$ & $75 \%$ & $\uparrow 0,3 \mathrm{~mm} \mathrm{Hg}$ \\
\hline & & Butterfly & $20 \mathrm{x} 1$ & $65 \%$ & $\uparrow 0,8 \mathrm{~mm} \mathrm{Hg}$ \\
\hline \multirow[t]{2}{*}{$\begin{array}{l}\text { Vieira et al., } \\
2006\end{array}$} & No dice & Press pecho & $4 \times 1$ & $80 \%$ & $\uparrow 4,4 \mathrm{~mm} \mathrm{Hg}$ \\
\hline & No dice & Handgrip & $2 \mathrm{~min}$. & & $\uparrow 4,1 \mathrm{~mm} \mathrm{Hg}$ \\
\hline
\end{tabular}

$1 \mathrm{mmHg}=$ milímetros de mercurio, $\mathrm{RM}=$ repetición máxima.

Ahora bien, la reducción de PIO está relacionada no solo con la reducción de la producción de humor acuoso, sino también con un mayor drenaje de este mismo, por lo cual este aumento del drenaje puede ser causado por ejercicio que provoque hiperventilación; esta razón podría ser una justificación en el ejercicio isométrico; sin embargo, dicha reducción es más significativa durante el ejercicio dinámico (Conte \& Scarpi, 2014).

Otra de las prácticas de actividad física que se están realizando en la actualidad es yoga. En el estudio de Dimitrova \& Trenceva (2017) se demuestra una disminución significativa en la PIO y, por otro lado, algunos estudios han encontrado aumentos en la PIO, después de ciertas posturas de yoga (Jasien, Jonas, de Moraes, \& Ritch, 2015).

Se menciona que las posturas en donde las personas están con la cabeza abajo (paradas de manos) o con los pies encima de la cabeza podrían provocar una elevación de la PIO, por un incremento de la irrigación coroidea y una mayor presión venosa epiescleral, en la cual el flujo de tejidos oculares se compromete. Ya que la coroides es un tejido muy vascularizado que puede causar cambios dinámicos en el líquido del humor 
acuoso, resulta un aumento de la PIO (Soares et al., 2015). Tal es el caso del estudio de Gallardo, Aggarwal, Cavanagh, \& Whitson (2006), que reporta un incremento de $10 \mathrm{~mm} \mathrm{Hg}$ en las personas, después de realizar una Sirsasana de yoga (cabeza hacia abajo). Mencionan que este tipo de prácticas son de un gran alto riesgo para personas con glaucoma.

\section{MÉTODo}

En el proceso de la selección de los artículos del estudio, se llevó a cabo una revisión de bibliografía en las siguientes bases de datos: SportDiscus con texto completo, psycINFO, Psychology and Behavioral Sciences Collection, Rehabilitation \& Sports Medicine Source, Gsoogle Académico, Research Gate, PubMed. Para hacer esta búsqueda de referencias se utilizaron la siguientes palabras claves: presión intraocular, glaucoma, ejercicio, actividad física.

En la Figura 3, se muestra el diagrama de flujo que se realizó durante el proceso de la revisión bibliográfica.

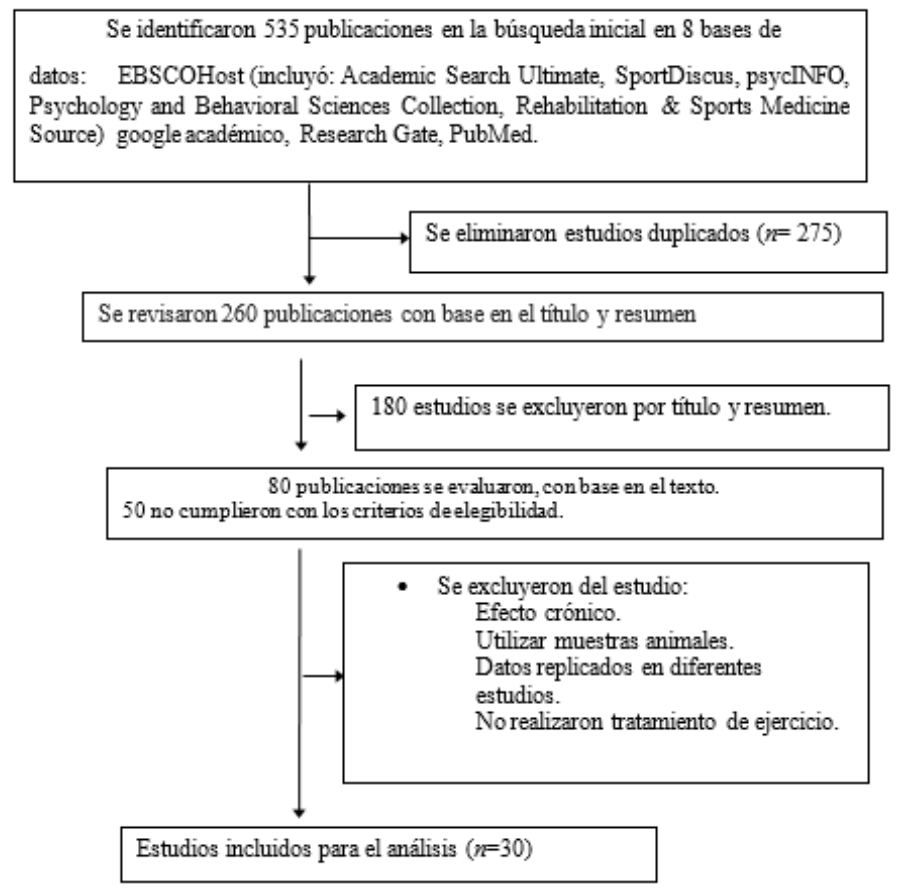

FIGURA 3.

Diagrama de flujo de los artículos seleccionados.

\section{Resultados}

La PIO alta es un factor de riesgo para la salud visual, especialmente porque puede ser la causante de presentar glaucoma, uno de los principales causantes de la ceguera a nivel mundial (Aspinall et al., 2008; Era et al., 2009; Leighton, 1972; Zhu et al., 2018). Estudios que datan desde los años setentas muestran los posibles efectos de la actividad física en los parámetros de la PIO (Leighton, 1972), estos pioneros brindaron las bases para ir esclareciendo conocimiento, así como el involucramiento de variables que podrían tener un efecto sobre la PIO (Dane et al., 2006). Con respecto al ejercicio aeróbico agudo, los resultados mostraron investigaciones con las variables que lo afectan: intensidad, duración, y tipo de ejercicio. Se encontró que las intensidades de ejercicio (bajas, medias y altas) ejercen efectos positivos, es decir, las intensidades que van desde el $40 \%$ hasta el $90 \%$ de la frecuencia cardiaca demuestran reducciones de la PIO (Conte \& Scarpi, 2014; Dane et al., 2006; Huang, 2015; Soares et al., 2015; Vera et al., 2017, 2018; Vieira, 2006); Del mismo 
modo, la duración del ejercicio es una variable que afecta la reducción de la PIO, estos cambios se dan, desde los cinco minutos de ejercicio continuo (Read \& Collins, 2011). Con respecto al tipo de ejercicio aeróbico, las investigaciones encontraron mejoras en diferentes modalidades como: bicicleta estacionaria, caminata, trote y correr (Ahmad, 1995).

El nivel de la actividad física es otra variable que afecta la PIO; se determinó que las personas sedentarias la reducen más, al comparar los cambios con personas físicamente activas. Por otro lado, se encontró que las personas con glaucoma lograron reducciones importantes en la PIO, después de realizar ejercicio aeróbico; sin embargo, los estudios realizados en esta población han sido muy pocos, por lo que no se podrían generalizar los resultados (Conte \& Scarpi, 2014; Dane et al., 2006; Huang, 2015; Soares et al., 2015; Vera et al., 2017).

En el caso del ejercicio contra resistencia, se demuestra que falta mucha investigación, hay resultados controversiales, como en: las afectaciones según la zona de trabajo (tren superior- tren inferior), ejercicios isométricos, posición del cuerpo, entre otras. Los resultados más contundentes parecieran ser los relacionados con la intensidad del ejercicio, estos presentan una relación directa, es decir, a mayor intensidad, mayor incremento en la PIO (Huang, 2015; Rüfer et al., 2014; Vera et al., 2018; Vieira, 2006), lo que muestra evidencia de la importancia de la supervisión o control en este tipo de ejercicio.

\section{Conclusiones}

Se puede mencionar que el ejercicio aeróbico agudo muestra un efecto significativo sobre la presión intraocular, ya que la disminuye; este resultado se mantiene en personas con diferentes niveles de actividad física (con un efecto más grande en personas sedentarias) así como también, en personas con diferentes condiciones visuales (patologías, visión normal).

Con respecto a la duración de ejercicio aeróbico agudo, es importante recalcar que cinco minutos de ejercicio continuo ejercen un estímulo en la PIO, lo que podría valorarse como una recomendación base, especialmente para personas que no están acostumbradas o se les dificulta la práctica del ejercicio.

La retención (efecto que se mantiene al finalizar el ejercicio) del ejercicio aeróbico sobre la PIO, se mantiene de manera proporcional al tiempo del ejercicio, es decir, si se realiza una caminata de 30 minutos, este mismo tiempo es el que dura la PIO en regresar a su nivel inicial; esto nos muestra una tendencia interesante que podría estudiarse con tratamientos de largos periodos para conocer el efecto crónico del ejercicio sobre la PIO.

Ahora bien, con respecto a los ejercicios de contra resistencia, se podrían citar los principales hallazgos:

- Los ejercicios en el tren superior del cuerpo aumentan PIO, comparándolos con los del tren inferior.

- Los ejercicios de alta intensidad aumentan la PIO.

- La información obtenida brinda evidencia científica en parámetros de este campo, por lo que se podrían ir construyendo recomendaciones con datos específicos para el beneficio de la población en general y especialmente en personas con PIO alta.

\section{RECOMENDACIONES}

Realizar investigaciones que analicen las variables de sexo y edad, como análisis de estudio y no como estadística descriptiva, para conocer el efecto del ejercicio sobre ellas.

- Investigar protocolos en donde el tratamiento involucre ejercicios de contra resistencia, para ir mostrando, con mayor claridad, la intensidad, el volumen, los tipos de ejercicios que afectan la PIO.

- Es importante que las investigaciones en este campo mejoren varios elementos como los diseños de investigación, principalmente, porque la mayoría no contaron con grupo control; muchas no especificaron la intensidad del ejercicio e incluso les faltó incluir aspectos descriptivos necesarios para poder contar con información más clara. 
- Involucrar en los estudios (con la supervisión de especialistas en el campo de la oftalmología) a personas con glaucoma, para saber si el ejercicio aeróbico podría contribuir en la salud visual.

- Al ser una temática en el ámbito de la salud y, por ende, con repercusiones en la afectación social, se recomienda continuar con el estudio de esta área.

\section{ReFERENCIAS}

Ahmad, I (1995). The effects of Mild, moderate and severe Exercise on intraocular Pressure in Glaucoma Patients. Japanese Journal of Physiology, 45, 561-569.

Ahmad, Rong, Bin y Dong (1996). Magnitude of decrease in intraocular pressure depends upon intensity of exercise. Korean J. Ophalmol, 10, 109-115.

Aspinall, P. A., Johnson, Z. K., Azuara-Blanco, A., Montarzino, A., Brice, R., \& Vickers, A. (2008). Evaluation of Quality of Life and Priorities of Patients with Glaucoma. Investigative Opthalmology \& Visual Science, 49(5), 1907. doi: 10.1167 /iovs.07-0559

Avunduk, A. M., Yilmaz, B., Şahin, N., Kapicioglu, Z., \& Dayanır, V. (1999). The Comparison of Intraocular Pressure Reductions after Isometric and Isokinetic Exercises in Normal Individuals. Ophthalmologica, 213(5), 290-294. doi: $10.1159 / 000027441$

Bakke, E. F., Hisdal, J., \& Semb, S. O. (2009). Intraocular Pressure Increases in Parallel with Systemic Blood Pressure during Isometric Exercise. Investigative Opthalmology \& Visual Science, 50(2), 760. doi: 10.1167/iovs.08-2508

Chong, C. W., Wang, S. B., Jain, N. S., Bank, C. S., Singh, R., Bank, A., ... Agar, A. (2016). Comparison between intraocular pressure spikes with water loading and postural change: WDT-ST study. Clinical \& Experimental Ophthalmology, 44(9), 768-775. doi: 10.1111/ceo.12777

Conte, M., \& Scarpi, M. J. (2014). A comparison of the intraocular pressure response between two different intensities and volumes of resistance training. Revista Brasileira de Oftalmologia, 73(1). doi: 10.5935/0034-7280.20140005

Dane, S., KoçEr, I., Demirel, H., üçOk, K., \& Tan, üNer. (2006). Effect of acute submaximal exercise on intraocular pressure in athletes and sedentary subjets. International Journal of Neuroscience, 116(10), 1207-1214. doi: $10.1080 / 00207450500516461$

Dimitrova, G., \& Trenceva, A. (2017). The short-term effect of yoga ocular exercise on intra-ocular pressure. Acta Ophthalmologica, 95(1), e81-e82. doi: 10.1111/aos.12850

Era, P., Pärssinen, O., Kallinen, M., \& Suominen, H. (2009). Effect of bicycle ergometer test on intraocular pressure in elderly athletes and controls. Acta Ophthalmologica, 71(3), 301-307. doi: 10.1111/j.1755-3768.1993.tb07139.x

Esfahani, M., Gharipour, M., \& Fesharakinia, H. (2017). Changes in intraocular pressure after exercise test. Oman Journal of Ophthalmology, 10(1), 17. doi: 10.4103/0974-620X.200689

Gallardo, M. J., Aggarwal, N., Cavanagh, H. D., \& Whitson, J. T. (2006). Progression of glaucoma associated with the Sirsasana (headstand) yoga posture. Advances in Therapy, 23(6), 921-925. doi: 10.1007/BF02850214

Harris, A., Malinovsky, V., \& MartinX, B. (1994). Correlates of acute exercise-induced ocular hypotension.,Invest Ophthalmol Vis Sci, 35(11), 3852-7

Huang, R. (2015). The Effect of Dynamic and Isometric Exercise on Refractive State, Accommodation and IntraOcular Pressure. Advances in Ophthalmology \& Visual System, 2(3). doi: 10.15406/aovs.2015.02.00047

Instituto Nacional de Estadística y Censos (Costa Rica) (Ed.). (2014). Costa Rica a la luz del Censo del 2011. San José, Costa Rica: Autor.

Jasien, J. V., Jonas, J. B., de Moraes, C. G., \& Ritch, R. (2015). Intraocular Pressure Rise in Subjects with and without Glaucoma during Four Common Yoga Positions. PLOS ONE, 10(12), e0144505. doi: 10.1371/ journal.pone.0144505

Karabatakis, V. E., Natsis, K. I., Chatzi balis, T. E., Lake, S. L., Bisbas, I. T., Kallinderis, K. A., \& Stangos, N. T. (2004). Correlating Intraocular Pressure, Blood Pressure, and Heart Rate Changes after Jogging. European Journal of Ophthalmology, 14(2), 117-122. doi: 10.1177/112067210401400206 
Kinoshita, T., Mori, J., Okuda, N., Imaizumi, H., Iwasaki, M., Shimizu, M., ... Mitamura, Y. (2016). Effects of Exercise on the Structure and Circulation of Choroid in Normal Eyes. PLOS ONE, 11(12), e0168336. doi: 10.1371/ journal.pone.0168336

Kozobolis, V. P., Detorakis, E. T., Konstas, A. G., Achtaropoulos, A. K., \& Diamandides, E. D. (2008). Retrobulbar blood flow and ophthalmic perfusion in maximum dynamic exercise. Clinical \& Experimental Ophthalmology, 36(2), 123-129. doi: 10.1111/j.1442-9071.2007.01646.x

Leighton, D. A. (1972). Effect of walking on the ocular tension in open-angle glaucoma. British Journal of Ophthalmology, 56(2), 126-130. doi: 10.1136/bjo.56.2.126

Martin, B., Harris, A., Hammel, T., \& Malinovsky, V. (1999). Mechanism of exercise-induced ocular hypotension, Invest Ophthalmol Vis Sci. 40(5):1011-5.

Okuno,T., Sugiyama, T., Kohyma M., kojima, S, Oku H \& Ikeda T (2006). Ocular blood flow changes after dynamic exercise in humans. Eye, 20,796-800.

Organización Mundial de la Salud. (s. f.). Ceguera y discapacidad visual. Recuperado de http://www.who.int/es/newsroom/fact-sheets/detail/blindness-and-visual-impairment

Ozmerdivenli, R., Simsek, E., Bulut, S., Karacabey, K., \& Saygin, O. (2006). Comparison of the effects of acute an regular exercise on intraocular pressure in turkish athlete and sedentarians. International Journal of Neuroscience, 116(3), 351-360. doi: 10.1080/00207450500442288

Read, S. A., \& Collins, M. J. (2011). The short-term influence of exercise on axial length and intraocular pressure. Eye, 25(6), 767-774. doi: 10.1038/eye.2011.54

Roddy, G. (2014). Reductions in Intraocular Pressure After Acute Aerobic Exercise: A Meta-Analysis. Clin J Sport Med, 24(5), 9.

Rüfer, F., Schiller, J., Klettner, A., Lanzl, I., Roider, J., \& Weisser, B. (2014). Comparison of the influence of aerobic and resistance exercise of the upper and lower limb on intraocular pressure. Acta Ophthalmologica, 92(3), 249-252. doi: 10.1111/aos.12051

Soares, A. S., Caldara, A. A., Storti, L. R., Teixeira, L. F. M., Terzariol, J. G. T., \& Conte, M. (2015). Variation of intraocular pressure in resistance exercise performed in two different positions. Revista Brasileira de Oftalmologia, 74(3). doi:10.5935/0034-7280.20150033

Vera, J., García-Ramos, A., Jiménez, R., \& Cárdenas, D. (2017). The acute effect of strength exercises at different intensities on intraocular pressure. Graefe's Archive for Clinical and Experimental Ophthalmology, 255(11), 2211-2217. doi: 10.1007/s00417-017-3735-5

Vera, J., Jiménez, R., Redondo, B., Cárdenas, D., \& García-Ramos, A. (2018). Fitness Level Modulates Intraocular Pressure Responses to Strength Exercises. Current Eye Research, 1-7. doi: 10.1080/02713683.2018.1431289

Vieira, G. M. (2006). Intraocular Pressure Variation During Weight Lifting. Archives of Ophthalmology, 124(9), 1251. doi: 10.1001/archopht.124.9.1251

Yan, X., Li, M., Song, Y., Guo, J., Zhao, Y., Chen, W., \& Zhang, H. (2016). Influence of Exercise on Intraocular Pressure, Schlemm's Canal, and the Trabecular Meshwork. Investigative Opthalmology \& Visual Science, 57(11), 4733. doi: $10.1167 /$ iovs.16-19475

Zhu, M. M., Lai, J. S. M., Choy, B. N. K., Shum, J. W. H., Lo, A. C. Y., Ng, A. L. K., ... So, K. F. (2018). Physical exercise and glaucoma: a review on the roles of physical exercise on intraocular pressure control, ocular blood flow regulation, neuroprotection and glaucoma-related mental health. Acta Ophthalmologica 96(6) doi: 10.1111/ aos. 13661

\section{BY-NC-ND}

